\title{
XXXVI. Ueber die Krystallisation des Rohrzuckers.
}

\author{
Von \\ L. Wulff in Schwerin in Meckl.
}

(Hierzu Tafel XI, Fig. 1-9.)

Von einer deutschen Rohrzuckerfabrik el'hielt ich vor kurzer Zeit eine Quantität ungewöhnlich entwickelten Zuckers. Ueber dieselbe schrieb man mir bei der Uebersendung :

"Die Krystalle befinden sich in grosser Anzahl in der Fullmasse unseres dritten Productes und haben sich grossentheils an den Wänden der circa hundertundfunfzig Hectoliter fassenden Fullmassenbehälter als feste Kruste angesetzl. Vor drei Jahren wurden die Krystalle hier schon gesehen, in den beiden darauf folgenden Jahren traten sie nicht auf. "

Es wird zum Verständniss nöthig sein, einige Angaben zu machen über die Producte, die für gewöhnlich gewonnen werden. Der Saft des ersten Productes wird im Vacuum eingekocht, bis er beginnt Krystalle zu bilden; und dann noch weiter eingedickt. Meist sind die gröberen der erzielten Krystalle nur ein bis zwei Millimeter lang; nur wenn der Zucker als Krystallzucker verkauft werden soll, werden die Krystalle zuweilen etwas grösser. Die Krystallisationsdauer beträgt nur wenige Stunden.

Der von dem ersten Producte abgeschleuderte Syrup wird eingedickt und erkaltet in grossen Kästen langsam im Laufe von ein bis zwei Wochen. Die Krystalle sind auch durchweg nur von der Grösse der Krystalle des ersten Productes und meist isolirt.

Der von dem zweiten Producle abgeschleuderte Syrup wird nochmals eingedickt und ergiebt dann beim langsamen Abkühlen auch nur höchtens ein bis zwei Millimeter grosse isolirte Krystalle; die Dauer der Krystallisation beträgt mehrere Monate, und dieselbe geht in grossen Kästen vor 
sich, die meist noch grösser sind, als oben für das dritte Product von Seiten der Fabrik angegeben war.

Anstatt der kleinen isolirten Krystalle sandte mir die Fabrik kleine Bruchstücke von Wandansätzen, die durchaus den Wandstücken von Candis ähnlich sind. Es fanden sich mehrfach Krystalle von anderthalb Centimeter Länge und einem Centimeter Breite, und gerade dieser auffalligen Grösse wegen waren mir die Krystalle gesandt worden. Bei den meisten organischen Verbindungen erhält man bei den Nachproducten kleine Krystalle, weil die zur Krystallisation nötbigen Strömungen durch die leichter löslichen Beimengungen gehemmt werden. Wenn sich nun hier bei unserem Vorkommen dennoch grosse Krystalle gebildet haben, so ist das nur so zu deuten, dass die für die Krystallisation günstigen Einflüsse, uber die bisher noch wenig Zuverlässiges bekannt ist, bei der vorliegenden Krystallisation so intensiv waren, dass trotz der Zähigkeit der Masse sich grosse Krystalle ausbilden konnten. Jedenfalls wurde es nicht angehen, die grossen Krystalle auf eine besondere Störung der Krystallisation zuruckzufübren. Es geschiebt zwar oft, dass auffallende Eigenschaften (z. B. Verzerrungen, Spannungserscheinungen) auf sogenannte Slörungen geschoben werden, uber deren Natur die Autoren der Phantasie ihrer Leser freiesten Spielraum lassen.

Die hier vorliegenden Krystalle sind in Bezug auf ihren Bau sehr normal, die Färbung der Grundmasse ist hellgelb bis fast glasklar. Der Umstand, dass die Hauptmasse der Krystalle wenig gefärbt ist, während Nachproductsäfte von dunkler Farbe sind, spricht für sehr langsames Wachsen. Ich weise auf diesen durchaus normalen Bau der Krystalle hier so nachdrücklich nur hin, um den Erklärungsversuch zu verhindern, dass hier besonders ungünstige störende Verhältnisse obgewaltet hätten. Wir müssen vielmehr annehmen, dass das vorliegende Vorkommen sich unter den möglichst günstigen Bedingungen gebildet hat.

Mir waren die Krystalle vor Allem der auffallenden Grössenentwickelung wegen gesandt, aber noch interessanter wurden sie mir bei Betrachtung der Flächenvertheilung, die an diesen Krystallen besonders auffällig ist.

Alle einfachen Krystalle stellen eine Combination der Flächen $a=$ $\{100\} \infty P \infty, d=\{10 \pi\}+P \infty, p=\{110\} \infty P$ dar, wie ich dieselben mehrfach zu beobachten Gelegenheit hatte $\left.{ }^{*}\right)$. Gewöhnlich zeigen die Krystalle mit der Fläche $d$ eine grosse Tendenz längliche Producte zu bilden, dies war bei den grösseren Krystallen meiner Muster nicht der Fall, wohl aber bei den kleineren, die sich nachträglich zwischen den grösseren gebildet hatten. Die analoge Beobachtung habe ich oft in Zuckersäften machen

*) Man siehe das Referat Seite $\mathbf{5 0 0 .}$ 
können, die von der Form des Candis abweichende Krystalle bildeten; die grösseren, welche sich zu Anfang in der warmen an Zucker reicheren Lösung gebildet hatten, waren der Normalform der Candiskrystalle viel ähnlicher als die kleinen nachträglich gebildeten, die sich in der Kälte in der schon theilweise auskrystallisirten Masse gebildet hatten, die ja verhältnissmässig unreiner ist.

An den mir gesandten Krystallen trat auch noch die Form $q\{011\}$ auf, welche beim Candis allgemein bekannt ist, und die ich auch an den Krystallen mil $d$ (ohne $c$ ) oft beobachtet habe. An fast allen Krystallen trat aber nur eine der Flächen von $q$ auf, wie es Fig. 1 Taf. XI darstellt.

Zuerst glaubte ich eine zufällige Erscheinung vor mir zu haben, bei näherer Betrachtung erwies sich aber das Feblen der einen Fläche $q$ als durchaus beständig für das gesammte Material. Die einzig vorhandene Fläche $q$ trat durchaus nicht in untergeordneter Ausbildung auf, sondern meist so wie es Fig. 1 zeigt, doch waren die Fälle, wo sie vorwiegend vor den Prismenflächen entwickelt war, jedenfalls weit häufiger, als das Vorkommen von kleinen Flächen $q$.

Als ich mich überzeugt hatte, dass es unzulässig sei, anzunehmen, dass bei der vorliegenden Krystallisation die Bedingungen für die Bildung der Flächen $q$ nur in unvollkommener Weise vorhanden gewesen seien, so dass nur an dem gunstiger gelegenen Ende der Kante von $\{110\}$ sich die Fläche $q$ hätte bilden können, suchte ich nach einer anderen Erklärung.

Bei der Untersuchung von Wandkrusten von verschiedenen Salzen hatte ich beobachtet, dass untergeordnete Flächen sich besonders leicht an gleich orientirten Krystallecken zeigen, wie dies ja zu erwarten steht mit Rücksicht auf die Strömungserscheinungen, die 0 . Le hmann mehrfach in dieser Zeitschrift beschrieben hat. Ich suchte mich also zu vergewissern, ob etwa alle einzelnen Flächen von $q$ gleich oder doch ähnlich orientirt seien. Die Krystallkrusten, welche ich erhielt, waren meist klein auseinandergebrochen, so dass ich mich über die Lage der Flächen an weiter von einander entfernten Krystallen nicht orientiren konnte.

Für viele der kleinen Bruchstucken was es auffallend, dass die einzelnen Flächen ähnlich orientirt waren, so dass man die Gruppen leicht so aufstellen konnte, dass alle Kanten von $\{110\}$ so lagen, dass die Kantenenden mit der einzelnen Fläche $q$ nach unten zu lagen. Bei den grösseren Mustern war dies aber nicht mehr der Fall, sondern man konnte nur für einzelne Partien der Krystalle eine analoge Lage von Einzelfächen erkennen, für benachbarte Krystallpartien lagen die Einzelflächen oft geradezu in entgegengesetzter Richtung. Auch bei einer Reihe von kleineren Gruppen fanden sich einzelne Krystalle, bei denen die Einzelfäche nicht so lag wie für die Gruppe. Es zeigte sich auch bei den grösseren Stlicken, 
dass sie leicht in kleinere Gruppen von ähnlich orientirten Krystallen zerbrachen.

Als ich die Hoflnung aufgeben musste, die Bildung der einzelnen Flächen auf etwaige Verticalströmungen in der Lösung zurückzuführen, untersuchte ich nochmals die Lage der Krystalle gegen einander, aber auch hierbei konnte ich keinerlei Anhalt erhalten. Theils treten die einzelnen Flächen an Exemplaren auf, die unmiltelbar an andere anstossen, theils an hervorspringenden Exemplaren, theils an Gruppen von parallel verwachsenen Krystallen. Gerade die Durchsicht der letzteren zeigte mir, dass nicht äussere Umstände die Bildung der einzelnen Flächen erzeugt hatten. Die parallel verwachsenen Krystalle zeigten die einzelnen Flächen gerade so deutlich an vorspringenden wie an einspringenden Partien.

Noch nach einer Ricbtung hin versuchte ich eine äussere, nicht im Wesen des Rohrzuckers begründete Erklärung für die einzelnen Flächen zu finden. Die Krystalle sind meist etwas verzerrt, weil sie dicht neben einander gewachsen sind. Die Prismenflächen sind oft ungleich, wie es Fig. 2 zeigt. Treten an einem solchen Krystalle die beiden Flächen von $q\{011\}$ auf, so musste eine lang und schmal und eine kurz auftreten. Es zeigte sich aber bei dem Durchsehen meines Materials, dass sowohl an langen Kanten $(\bar{T} T 0):(10 \bar{T})$ wie an den spitzen Ecken von drei benachbarten Flächen $(100)(1 \bar{T} 0)(10 T)$ die einzelnen Flächen vorkommen.

So gern ich also auch beim Zucker, so wie ich es fruher beim Alaun gethan habe, die scheinbare Tetartoëdrie durch äussere Wachsthumsbedingungen erklärt bälte ${ }^{*}$ ), die vorliegenden theilweise für Zucker recht scharfkantig entwickelten Krystalle nöthigten mich, die in Fig. 1 dargestellte Flächencombination als die typische wenigstens für das vorliegende Zuckervorkommniss anzuerkennen.

Muss dies anerkannt werden, so tritt vorerst uns die Frage entgegen: Ist der vorliegende Zucker eine andere Modification des Zuckers, oder tritt hier nur die sonst versteckle Flächendifferenzirung des Zuckers hervor. Die Annabme einer zweiten bisher nicht beobachteten Modification des Zuckers wäre verfehlt. Die Krystalle zeigen sonst durchaus die Eigenschaften der Zuckerkrystalle, worauf ich unten noch weiter eingehen werde. Tritt ein Körper in mehreren Modificationen auf, so ist das Auftreten neuer nicht beobachteter Modificationen stets bei besonders hohen oder niedrigen Temperaturen zu erwarten, wäbrend die vorliegenden Nachproductzucker sich gerade bei mittleren Temperaturen gebildet haben. Ich habe oft aus $100^{\circ}$ heissen Zuckerlösungen und bei strenger Kälte Zuckerkrystallisationen vorgenommen und stets den gleichen Zucker erhalten, bis auf einige matte Krystallvorkommnisse in uberhitzten Zuckerlösungen.

*) Diese Zeitschr. 5, 81 . 
Es bleibt also nur die Annahme, dass bei dem vorliegenden Material die Flächendifferenzirung vollkommen auftrete, die sonst versteckt ist. Dass sich gerade bei diesen Krystallen die typische Krystallform entwickelt hat, ist nicht auffällig, denn jedenfalls sind die Bedingungen, unter denen sich die Krystalle gebildet haben, die denkbar günstigsten gewesen, da in den schlechten. Säften mit geringer Krystallisationstendenz sich Candiskrystalle bilden konnten. Bei der Candikrystallisation sind zwar auch jedenfalls sehr günstige Krystallisationsverhältnisse anzunehmen, doch ist es ja eine für viele Substanzen beobachtete Thatsache, dass gerade an Krystallen aus unreinen Lösungen leicht die bei reinen Lösungen nicht beobachteten Flächendifferenzirungen auftreten.

Wollen wir das Auftreten der einzelnen Fläche krystallographisch näher bezeichnen, so kann dies in zweierlei Weise geschehen, je nachdem man den Zucker im monoklinen System belässt, wo er bisher unbestritten seinen Platz gehabt, oder ihn ins trikline System stellt.

Ist der Hohrzucker monoklin, so müssen wir ihn als tetartoëdrisch ansehen. In meiner Arbeit über die Hemiëdrien und Tetartoëdrien*) habe ich ausgefuhrt, dass vom geometrischen Gesichtspunkte zwei Darstellungsweisen der Flächendifferenzirung im monoklinen Systeme als gleichberechtigt anzusehen seien, und dass nach der einen Darstellungsweise auch Tetartoëdrie möglich. sei, und dass das Tetartoëder eine einzelne Fläche sei. Die drei Hemiëdrien bilden eine Triade $\left.{ }^{* *}\right)$.

In Fig. $3 a, b, c$ Taf. II sind auf den vier Flächen einer monoklinen Hemipyramide die drei Arten der hemiëdrischen Flächendifferenzirungen angegeben. Statt der naturgemässen Aufstellung der Krystalle mit der Orthodiagonale als Verticalaxe habe ich auch die allgemein übliche Aufstellung in den Figuren angewandt, aber trotzdem für die hemimorphen Hälften die

*) Diese Zeitschr. 13, 499.

**) Ich will hier nicht unerwähnt lassen, dass ich nach Publication meiner Arbeiten im vorigen Bande von Herrn B. von Kolenko die russische Bearbeitung der Publication desselben Autors in dieser Zeitschr. 9, 1 erhielt. Aus den derselben beigefüglen übersetzten Stellen ergiebt sich, dass die russische Bearbeitung wesentlich eingehender auf die Ableitung der Hemiëdrien und Tetartoëdrien eingeht.- Der Verf. hat für mehrere Tetartoëdrien die Hemiëdrien zu Triaden vereinigt und wäre vielleicht zu meiner einheitlichen Ableitung gekommen, wenn er bei der rhomboëdrischen und trigonotypen Hemiëdrie je zwei Unterarten angenommen hätle. Dann hätte er auch nicht (S. 63) abgeleitet, dass die Combination der rhomboëdrischen (II) und hemimorphen (V) Hemiëdrien identisch sei mit der Combination der deuterotrigonolypen (IV) und hemimorphen (V) Hemiëdrie. So stellt er S. 61 für seine Combinalion die Behauptung auf $" I+I V \doteq I I+I V *$ und $S .63 » I I+V=I V+V$ ", was mit einer stricten Ableitung nach Triaden nicht vereinbar ist, denn 1 bezeichnet die trapezoëdrische Hemiëdrie. Jedenfalls ist v. Kolenko der allgemeinen Ableitung nach Triaden aber wesentlich năher gekommen, als ich bei meiner Publication glaubte, dass dies geschehen sei. 
Zeichen $o$ (oben) und $u$ (unten) angewandt, wie für die anderen Krystallsysleme in meiner oben citirten Arbeit.

In Fig. $3 a$ ist die Hemipyramide als hemimorph differenzirt dargestellt.

In Fig. $3 b$ ist die Hemipyramide nach der triklinen Hemiëdrie halbirt. Die Flächen sind mit [+] und [-] bezeichnet. Wie aus der Figur ersichtlicb, ist das positive Hemiëder mit dem negativen zur Deckung zu bringen, wenn man den Axencomplex um die Orthodiagonale um $180^{\circ}$ dreht.

In Fig. $3 c$ ist die Hemipyramide so halbirt, dass die Hemimorphierichlung eine Richtung des Hauptschnittes ist. Die beiden Hälften sind mit $p$ und $n$ bezeichnet, und ist aus der Figur leicht ersichtlich, dass diese Hemiëder nicht wie die Hemiëder nach der orthodiagonalen Hemimorphie enantiomorph sind, sondern wie bei der triklinen Hemiëdrie mit einander zur Deckung gebracht werden können durch eine Drehung von $180^{\circ}$ um die Orthodiagonale.

In Fig. 4 ist die Hemipyramide gezeichnet und mit allen drei hemiëdrischen Bezeichnungen versehen. Wir haben vier verschieden bezeichnete Flächen, von denen jede mit einer der anderen zur Deckung gebracht werden kann. Wir haben hier demnach vier einzelne Flächen, die wie die mehrflächigen Tetartoëder zwei enantimorphe Paare bilden.

II. Ist der Rohrzucker triklin, so würde damit nachgewiesen sein, dass trikline Substanzen nicht bloss morphologisch, sondern auch elektrisch hemimorph sein können, denn die Hemimorphie des Rohrzuckers ist sowohl in Anbetracht der Flächenentwickelung und Wachsthumsverhälınisse, als auch der Pyroëlektricität ausser allem Zweifel.

Die Doppelfläche des triklinen Systemes würde bei der Entwickelung einer Hemimorphie nach irgend einer Richtung, die derselben nicht parallel ist, in zwei singuläre Flächen zerfallen, die für sich zwei enantiomorphe Hemiëder darstellen würden. Man müsste also auch rechte und linke Krystalle im triklinen Systeme unterscheiden können.

Es sind bereits an einigen triklinen Substanzen Beobachtungen daruber gemacht, dass einzelne Flächen vereinzelt auftreten, nicht als parallele Doppelflächen. V. von Ze pharovich hat vom essigsauren-salpetersauren Strontian derartige Krystalle beschrieben. In seinen Figuren finden sich neben holoëdrischen*) auch hemièdrische und zwar enantiomorphe Gestalten. Ich gebe die Fig. 3. und 4 des Autors auf Taf. XI verkleinert wieder in Fig. 5 und 6 . Bei den Figuren sind die nicht an beiden Krystallen auftretenden Flächen des Autors nicht mitgezeichnet, um beide Figuren vollkommen analog zu haben. Im Text geht der Verf. nicht näber auf die Bedeutung der Flächendifferenzirungen ein.

*) Sitzber. d. k. Akad. der Wiss. Wien. 41, 517, Fig. 2. 
Auf die Frage nach dem System des Zuckers komme ich weiler unten zurück.

Neben den beschriebenen Exemplaren mit einer Fläche $\{011\}$ waren sehr viele Krystalle vorhanden, welche die Fläche gar nichl hatten. Die betreffenden Krystalle können dreierlei Art sein.

Erstens können es Krystalle sein, von denen allein das rechte Ende frei entwickelt ist. Bei Ansätzen an Wänden plegen allerdings die Krystalle mit dem rechten Ende aufgewachsen zu sein, aber die hervorragenden Exemplare waren meist nicht unmittelbar an der Wandung angewachsen, sondern die betreffenden Krystalle stellten die obersten Individuen einer Schicht von Krystallen dar, wie man sie meist auch an Wandstücken beim Candis beobachten kann. So ist jedenfalls zu erwarten, dass sich auch Krystalle mit frei entwickeltem rechten Ende vorfinden. Zweitens könnten es Exemplare sein, an denen die Flächen $q$ nicht entwickelt sind. Drittens könnten die betreffenden Krystalle Zwillinge sein. Es sind ja beim Zucker lange Zwillinge bekannt, bei denen die gleichen Pole einander zugewandt sind, so dass also die Orthodiagonalen parallel und zwar entgegengesetzt gerichtet sind, während die verticale Axe gleichgerichtet in beiden Individuen ist, und die Klinodiagonalen beider Individuen nicht parallel sind. Auf diese Zwillinge komme ich noch weiler unten zuruck. Es können aber auch Zwillinge vorkommen, bei denen alle Axen der beiden Individuen parallel sind. Drehen wir nämlich einen Zuckerkrystall mit einer singulären Fläche um $180^{\circ} \mathrm{um}$ die Orthodiagonale, so fallen zwar die Axen wieder zusammen, aber die singuläre Fläche hat jetzt eine neue Lage.

Weil die Zuckerkrystalle meist mit einer getrockneten Syrupschicht bedeckt sind, ist es schwer, die Zwillinge mit lauter parallelen gleich gerichteten Axen als solche zu erkennen, da die $Z$ willingsnähte verdeckt werden. Bei einzelnen Exemplaren konnte aber die Zwillingsnaht gut verfolgt werden, auch zeigten sich an den Zwillingsnähten bei einigen Exemplaren kleine Flächen von $q$. Am deutlichsten trat die Zwillingsbildung hervor an dem in Fig. 7 dargestellten Exemplare. Das längere, in Figur rechte, Exemplar ragte weit uber das zweite Exemplar hervor und zeigte eine stark entwickelte Fläche $q$, das kürzere Exemplar zeigte keine singuläre Fläche, wie dies auch sein muss, da das Ende, an dem $q$ auftreten musste, nicht frei entwickelt ist. Sind die Einzelkrystalle gleich gross, so können leicht scheinbare Individuen ohne $q$ entstehen.

Wiederholt sich die Zwillingsbildung nach dieser Verwachsungsart, indem mit einem mittleren Individuum zu beiden Seiten Zwillingskrystalle verwacbsen, so werden die äusseren Individuen parallel. Ein sehr klarer glatuflächiger Krystall war als Drilling von dieser Bildungsweise deutlich zu 
erkennen. Auch fanden sich mehrere Gruppen, in denen die Zwillingsbildung mehifach wiederholt war.

Ganz vereinzelt fanden sich Krystalle mit zwei Flächen von $q$. An einzelnen Krystallen fanden sich zweimal die Flächen in doppelter Anzahl. Bei einer Gruppe zeiglen sich mehrere Krystalle mil je zwei Flächen $q$. Die Krystalle können in zweifacher Weise gedeutet werden. Entweder tritt an ihnen die meist nicht entwickelte Fläche von $\{011\}$ auf (wie es ja nicht gerade selten ist, dass man an einzelnen Krystallen Flächen findet, die sich bei der Mehrzahl derselben nicht zeigen) oder es sind die Krystalle Zwillinge, bei denen die Enden der Kante von $\{110\}$, welche keine Fläche $q$ haben, zugewandt sind, dann müssen sich natürlich an den beiden abgewandten äusseren Enden die beiden einzelnen Flächen zeigen. Bei einigen Exemplaren war die Zwillingsbildung deutlich zu erkennen, ich bin aber auch für die andern geneigt, anzunehmen, dass es Zwillinge sind. Wie bereits angegeben, ist es schwer, an Nähten die Zwillingsnatur sicher zu erkennen.

Die Krystalle mit mehreren Flächen $q$ lassen sich, so weit die Zwillingsnatur deutlich ersichtlich ist, als Drillinge oder Viellinge erkennen. Am deutlichsten war der Drilling entwickelt, den Fig. 8 von links her gesehen darstellt. Das miltlere Individuum war am stärksten entwickelt und zeigt $p\{110\}$ und $q\{011\}$, das in der Figur rechts befindliche Exemplar ist nur durch eine Naht geschieden und zeigt gleichfalls $\{110\}$ und $\{0 / 1\}$. Das in Fig. 8 links befindliche Exemplar ist nur klein und zeigt keine Fläche $q$, wie dies ja auch sein muss, da das Ende, an dem die Fläche auftreten wurde, nicht frei entwickelt ist.

Gerade in diesen eben beschriebenen Zwillingsbildungen documenlirt es sich, dass wir es bei diesem Zuckervorkommen mit einem typischen zu thun haben, und waren es gerade die Zwillinge, welche mir die letzten Scrupel nahmen. Es können solche Zwillinge nur an dem hier beschriebenen monoklin-tetartoëdrisch erscheinenden Vorkommen äusserlich erkannt werden.

Die Zwillinge mit enlgegengeselzt gerichteter Orthodiagonale waren durchaus nicht selten und zeigten manche Eigenschaften der fruher *) von mir beschriebenen. Dieselben zeigen meist die an Flächen armen Pole einander zugewandt und waren wesentlich flacher als die Einzelkrystalle. Auffallend war das Auftreten der Flächen $\{001\}$ an diesen Zwillingen. Einzelkrystalle zeigten diese Form gar nicht. Sowohl die Formen $\{001\}$ als $\{10 T\}$ waren wenig ebenflächig, wie ich auch sonst an den Zuckerzwillingen nit enlgegengesetzt gerichteten Polen beobachtete. Auffallend war der Zusammenhang zwischen den Flächen $\{001\}$ eines Exemplares und $\{10 \bar{T}\}$ des dazu in Zwillingsslellung befindlichen. Dieselben gehen allmäh-

*) Man sehe das Referat Seite 500. 
lich in einander über, und siebt man an der Gestalt der Flächen $\{001\}$ deutlich, dass es Flächen sind, welche den einzelnen Krystallen durch die Nachbarschaft des anderen Individuums aufgedrungen sind. Dieselben sind nämlich selten gleich breit, sondern verschmälern sich in beiden Individuen in entgegengesetzter Richtung. In Fig. 9 isl eine Ansicht eines Zwillings in doppelter Grösse so dargestellt, dass die Projectionsebene die Symmetrieebene der monoklinen Holoëdrie ist. Die Figur stellt auch zugleich die etwas auffallende Art der Zwillingsverwachsung dar, die statt hatte, wenn die Nähte beiderseits gleich lagen. Die Hälfte aller Zwillinge halle die eben angegebene Form, so dass die Individuen nur mit einer dünnen Schicht verbunden waren. Wir haben es hier jedenfalls mit Krystallen zu thun, welche zu den $Z$ willingen gehören, die nicht durch blosse Juxtaposition gewachsen sein können*). Die Zwillinge von anderer Form näherten sich in ibrem Baue häufig dem der gewöhnlichen Candiszwillinge, meist war deutlich ersichtlich, dass die $Z$ willinge nicht aus zwei einfachen Individuen bestanden, sondern dass die beiden Theilindividuen selbst wieder aus Individuen bestanden, welche in Zwillingsstellung waren nach Fig. 7 und 8.

Nach Beendigung der Untersuchung erhielt ich von derselben Fabrik nochmals abnorm grosse Individuen, doch waren dieselben nicht ganz so gross, wie die Krystalle der ersten Sendung. Die Fläche $q$ war auch an vielen Individuen einzeln entwickelt, doch waren auch Krystalle gar nicht selten, an denen die zweite Fläche klein entwickelt war, so dass die Krystalle einen Uebergang zu den gewöhnlichen Zuckerkrystallen darstellen. Auch die verschiedenen Zwillingsverwachsungen von Fig. 7, 8 und 9 fanden sich vor. Da die Krystalle kleiner waren, so waren auf den kleinen Bruchstucken mehr Krystalle vereinigt, als auf den Bruchstücken des ersten Vorkommens und konnte ich mich nochmals davon überzeugen, dass die vorwiegenden Flächen von $\{011\}$ in den verschiedensten Lagen der Krystalle vorkommen, und dass auch bei diesen Gruppen keine andere Erklärung möglich ist als die vorher gegebene, dass das Auftreten von $q$ als einer einzelnen Fläche durchaus typischer Natur ist.

Als ich Versuche anstellte, die Krystalle, die Fig. 1 darstellt, in reinen Zuckerlösungen weiter wachsen zu lassen, stellte sich sofort die zweite Fläche von $\{011\}$ ein, auch $\{001\}$ trat sofort auf, nachdem die Krystalle eine Stunde gewachsen waren. Zwillinge nach Fig. 7 und 8 verhielten sich ebenso, so dass beim Weilerwachsen der Unterschied der verschiedenen Gestalt ganz schwand. Hiernach durfte man erwarten, dass auch die gewöbnlichen als Individuen bezeichneten Zuckerkrystalle theils Individuen sind, theils Zwillinge nach Fig. 7 und 8, deren Zwillingsnatur man morphologisch an der äusseren Gestaltung nicht nachweisen kann.

*) Vergl. diese Zeitschr. 10, 374. 
tch habe meine verschiedenen Muster von Zuckerkrystallen durchgeseben und dabei gefunden, dass auch bei Krystallen, die von klein aul in Bewegung gewachsen sind, das Grössenverhialtniss der Flachen $q$ sehwankender ist, als man erwarten sollte, wenn beide Flächen von gleicher Bedeutung wären. Um aber für die verschiedenen flächenreichen Krystalle die Ëinfachheit oder den Zwillingsaufbau nachzuweisen, gehören noch umfangreiche Züchtungsversuche.

Ich hatle zu Anfang es unentschieden gelassen, ob man den Zucker als monoklin-tetartoëdrisch oder triklin-hemimorph ansehen wolle. Eine bestimmte Entscheidung auf diese Frage kann auch hier nicht gegeben werden, aber nach dem Fortwachsen der Krystalle zu urtheilen, sowie nach dem Verhalten des reichen Zwillingsmaterials liegt kein Grund vor, zum triklinen System überzugehen, sondern es ist vielmehr vorerst anzunebmen, dass der Zucker monoklin sei, wie bisher angenommen wurde.

Zwecks optischer Untersuchung und zu Aetzungsversuchen habe ich einen Theil meines Materials zwei Fachgenossen überlassen, da ich selbst nicht Gelegenheit habe, die betreffenden Untersuchungen auszuführen.

Ich hatte mir schon vor Jabren Zuckermaterial krystallisirt, um die $\mathbf{A n -}$ gaben uber die Winkelwerthe des Zuckers zu revidiren, doch halle ich damals nicht Gelegenheit, mein Material frisch zu untersuchen, und die Spiegelung der Zuckerkrystalle verliert bald an Güte. Ich stelle gern von meinem reichhaltigen Material zur Verfügung, wenn ein Fachmann goniometrische Untersuchungen unternehmen will, um den Versuch zu machen, die Winkel des Zuckers zu bestimmen, uber welche bisher die Angaben zum Theil sehr variiren.

Aussichtslos ist eine solche Untersuchung keineswegs. Auch für essigsaures-salpelersaures Strontian ward erst von V. von Z e pharavich eine geringe, aber constante Abweichung von $90^{\circ}$ constatirt. Wenn bei einer solchen Untersuchung das genannte Salz zum Vergleich herbeigezogen würde, so würde man jedenfalls ein reiches und mannigfaltiges Material von Zwillingen finden, die scheinbar monoklin sind und manche Analogien mit den Zuckervorkommnissen erkennen lassen. Ich habe die Krystallisation des Salzes nur so weit verfolgt, dass ich die Hemimorphie der Salze als typisch erkannte.

Weit ausgeprägter als beim salpetersauren-essigsauren Strontian ist die hemiëdrische Entwickelung der triklinen Bitartrate des Strontians, von denen A. Scacchi*) zwei Hydrate als triklin-hemiëdrisch beschreibt. Es treten sehr verschiedene Flächencombinationen auf, doch ist aus den Figuren nicht zu ersehen, dass enantiomorphe Combinationen beobachtet sind, die

*) Tartrali di strontiana e di barite. Atti della R. Accademia delle Scienze, Fisiche e Matematiche. Vol. 1.

Groth, Zeitschrift f. Krystallogr. XIV. 
man ja auch erst bei den Krystallen der en tsprechenden Salze als Linksweinsäure erwarten sollte. Während beim salpetersauren-essigsauren Strontian sich eine Richtung bei der Zwillingsbildung als die Axe der Hemimorphie zu erkennen giebt, lässt sich aus den bisher vorliegenden Untersuchungen uber das Bitartrat des Strontians nicht mit Sicherheit die Hemimorphieaxe erkennen.

Jedenfalls ist es durchaus nicht gerechtfertigt, dass die Hemiëdrie des triklinen Systemes in einer Reihe von Lehrbüchern ubergangen oder gar negirt wird. Die triklin-hemiëdrischen Salze stellen die niedrigste Stufe der Flächenentwickelung dar und würden jedenfalls ein reiches Beobachtungsmaterial für eine eingehende morphologische Monographie dieser bisher vernachlässigten Gruppe bielen. 